\title{
Two-stage DEA for Bank Efficiency Evaluation Considering Shared Input and Unexpected Output Factors
}

\author{
Song Aifeng ${ }^{1}$, Zhang XiaoYang ${ }^{2}$, Huang Weilai ${ }^{1}$, Yangxue ${ }^{3}$, Yang Juan ${ }^{3 *}$ \\ ${ }^{1}$ School of Management, Huazhong University of Science and Technology, Wuhan,450074 \\ ${ }^{2}$ School of Business, ZhengZhou Vocational College of Finance and Taxation, Zhengzhou, 450048 \\ ${ }^{3}$ School of Management and Economics, North China University of Water Resources and Electric Power, Zhengzhou ,450046.
}

\begin{abstract}
With the increasingly fierce market competition, only by relying on high-quality products and high customer satisfaction can enterprises survive in the fierce competition. Among many evaluation methods, Data Envelopment Analysis (DEA), as a non-parametric statistical method to effectively deal with multi-input and multi-output problems, has received more and more attention in evaluating the relative efficiency of decision-making units. In the process of bank efficiency evaluation based on DEA method, there will be a situation that banks have both dual role factors and unexpected output factors. The Two-stage DEA model provides an effective analysis method to solve the problem of bank efficiency evaluation of complex organizational structure. In order to evaluate the efficiency of unexpected output with uncertain information, a stochastic DEA model of unexpected output is established.
\end{abstract}

\section{Introduction}

With the increasingly fierce market competition, only by relying on high-quality products and high customer satisfaction can enterprises survive in the fierce competition. The evaluation and selection of bank efficiency is a process of selecting the bank with the best efficiency by comprehensively comparing the capital turnover and customer satisfaction of the bank, which is a typical multi-objective and multi-criteria decision-making problem [1]. Whether it is an enterprise as a node in the supply chain or a non-profit organization such as government, hospital and school, it is highly dependent on the success of the bank efficiency evaluation and selection process [2]. In reality, the value of bank efficiency evaluation criteria may not only be accurate quantitative value, but also inaccurate estimation value, ordinal value and language variable value [3]. Due to the extreme importance of bank efficiency evaluation and selection, relevant decisions have attracted extensive attention and research. Among many evaluation methods, Data Envelopment Analysis (DEA), as a non-parametric statistical method for effectively dealing with multi-input and multi-output problems, has received more and more attention in evaluating the relative efficiency of decision-making units [4]. The traditional DEA models are all based on deterministic problems, but in actual life, the interference of factors such as measurement and randomness of economic laws will produce inaccurate data problems.

Bank efficiency has an important impact on the performance of core enterprises and the entire supply chain, so it is necessary to establish an efficient and realistic model of bank efficiency evaluation and selection. Data envelopment analysis, as a non-parametric statistical method to effectively deal with multi-input and multi-output problems, is suitable for the same type of effectiveness evaluation with multiple inputs and outputs, and has outstanding advantages in solving bank selection problems [5]. Because fuzzy mathematics has the advantage of mathematically expressing uncertainty and ambiguity in generating decisions using approximate and uncertain information, and provides many formal tools with inherent inaccuracies, so in dealing with fuzzy index values On the issue of bank efficiency evaluation, fuzzy DEA has unparalleled advantages [6]. The advantages of DEA in the evaluation of bank efficiency are obvious. It can process multiple indicators at the same time without the need to give weights in advance, especially after the evaluation, the direction of bank improvement can be obtained by projection [7]. When using DEA to evaluate bank efficiency, it is necessary to first determine the input elements and output elements. Although the positioning of some elements is obvious, it is confusing when determining whether some elements are input or output [8]. The two-stage DEA model provides an effective analysis method for solving the problem of bank efficiency evaluation of complex organizational structures.

\section{DEA Model Introduction and Efficiency Evaluation}

In the process of production practice, producers of course want to use as little input as possible and get as much

${ }^{*}$ Corresponding author: Yang Juan 
output as possible. As long as there are dual role elements in the system, the system can certainly be decomposed into multiple subsystems. In the use of DEA method, it is generally assumed that the less input, the better output, which in fact assumes that all outputs are expected by decision makers. In actual production activities, input and output have different positions and functions. In order to make comparison and evaluation, input data and output data must be integrated. Therefore, an analysis and evaluation method that can effectively integrate input and output data needs to be found. The production system containing dual role elements is decomposed into a plurality of subsystems, wherein the dual role elements are intermediate variables between the two subsystems. In the actual multi-stage complex network production process, unexpected outputs such as environmental pollutants often occur [9]. At this time, the technology that can simultaneously produce more expected output and less unexpected output with relatively less resource input can be used as a criterion to measure whether the production decision-making unit is effective. In the traditional DEA model, since all outputs are actually assumed to be expected outputs, the ineffectiveness of the evaluated decision unit (DMU) may come from two aspects.

The traditional DEA model does not consider the internal structure of DMU and regards the whole DMU as a black box, while the two-stage DEA model tries to depict the two-stage system and find the relationship between the system and its subsystems. DEA's efficiency evaluation idea is to achieve as much output as possible with as little input as possible, and on this basis to evaluate the relative effectiveness of all decision-making units. Assuming that there are $\mathrm{n}$ decision units, their operations can be divided into two phases or processes, as shown in Fig. 1.

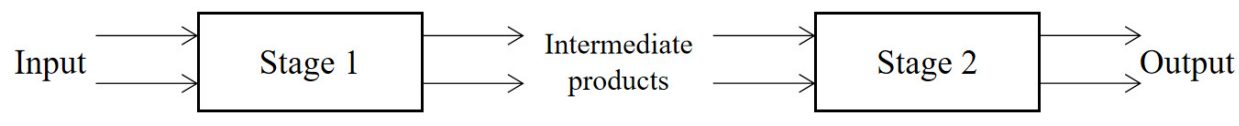

Fig. 1 Two-stage system

Suppose the production system to be processed has $n$ decision-making unit DMUs, and each DMU has 3 input-output vectors. Respectively defined as vector matrices: $X=\left(x_{i j}\right) \in R^{m \times n}, Y^{g}=\left(y_{i j}^{g}\right) \in R^{s 1 \times n}$ and $Y^{b}=\left(y_{i j}^{b}\right) \in R^{s 2 \times n}, \mathrm{X}>0, \mathrm{Y}^{\mathrm{g}}>0, \mathrm{Y}^{\mathrm{b}}>0$, the production possibility set $\mathrm{P}$ can be defined as:

$$
P=\left\{\left(x, y^{g}, y^{b}\right) \mid x \geq X \lambda, y^{g} \geq Y^{g} \lambda, y^{b} \geq Y^{b} \lambda, \lambda \geq 0\right\}(1)
$$

The SBM model with undesired output is:

$$
\min \rho=\frac{1-\frac{1}{m} \sum_{i=1}^{m} \frac{S_{i}^{-}}{x_{i 0}}}{1+\frac{1}{S_{1}+S_{2}}\left(\sum_{r=1}^{s 1} \frac{S_{y}^{g}}{y_{r 0}^{g}}+\sum_{r=1}^{s 2} \frac{S_{r}^{b}}{y_{r 0}^{b}}\right)}
$$

Among them, $S$ is a relaxation variable, $\lambda$ is a weight vector, and the objective function value $\rho$ is strictly decreasing with respect to $\mathrm{s}$.

In the actual production practice, you will find that the pursuit of expected output is accompanied by the production of some undesirable by-products. Of course, policymakers can simply ignore these factors when evaluating bank efficiency, but the results will be very different from the facts. During the application of the DEA method, if there are both expected output and undesired output, the processing should be different. Only after decomposing and applying the two-stage DEA method to solve the efficiency of the entire system and the efficiency of the subsystems can the efficiency of the system containing dual role elements be measured reasonably and correctly. The research of the DEA model is mainly focused on the evaluation ranking of the input and output indicators and their relative efficiency, so that the efficiency value of multiple decision-making units is inevitably. Under the DEA framework, there are many ways to deal with undesired output, such as taking undesired output as input, or performing monotonous decreasing conversion on undesired output, and then using the converted variable as output, and direction distance function method, etc.

\section{DEA Model for Evaluating Bank Efficiency with Unexpected Output}

Unexpected output exists in the bank's selection process. Only by minimizing the unexpected output can the optimal benefit of producing as many expected outputs as possible with the least input be satisfied. There are two ways to solve the problem when there are multiple effective decision units in DEA model and all DMUs need to be fully sorted. In the new model, the decision unit can be effective as a whole only if and only if both phases are effective. The unexpected output factor is introduced into the SBM model, and the unexpected output SBM model is proposed, which not only solves the slack problem of input and output, but also solves the efficiency evaluation problem under unexpected output. Finally, the model is applied to the bank efficiency evaluation of a company.

In the actual production process, people often expect less input and unexpected output in each stage or department. The more expected output is, the better. Each department reasonably allocates resources according to its importance, so as to achieve the improvement of the overall efficiency of the decision-making unit. The whole production process is divided into $\mathrm{k}$ stages. In the $\mathrm{k}$ stage, mk inputs $x_{i j}^{k}\left(i=1,2, \ldots m_{k}\right)$ produce $\mathrm{Rk}$ expected outputs $y_{r j}^{k}\left(r=1,2, \ldots, R_{k}\right)$ and Pk unexpected outputs $u_{p j}^{k}\left(p=1,2, \ldots P_{k}\right)$

defined as $\lambda_{j}^{k}(j=1, \ldots, n)$, and the production 
possibility set under the premise of constant production scale returns can be expressed as:

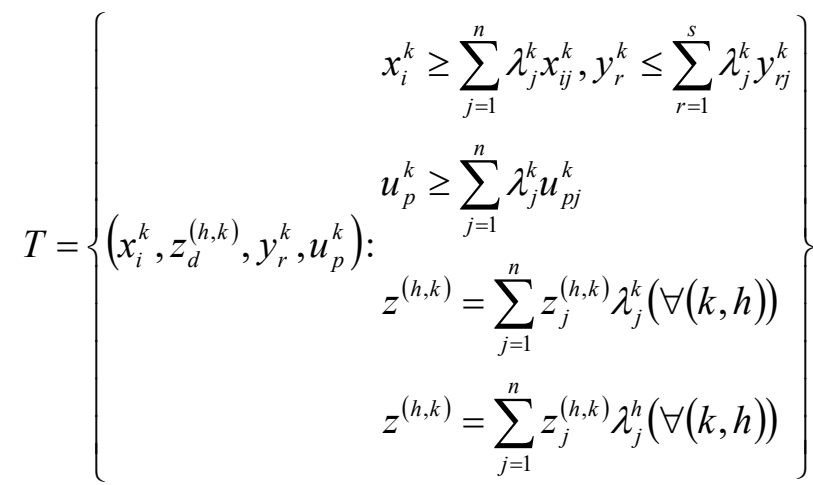

Because the performance of the whole production process of the decision-making unit is the result of the two-stage performance synthesis, the model can decompose the overall efficiency into the two-stage efficiency, which can help the management or decision-makers identify the stage where the root cause of the overall inefficiency occurs, thus taking targeted improvement and improvement measures to enhance the efficiency of the stage. For multi-variety and multi-customer production enterprises, in order to meet the needs of different customers, the enterprises must make corresponding inventories, distribute goods according to the requirements of customers for variety and quantity, and deliver them to the designated place at the designated time [10]. When the input or output is interval value, the DEA method used is called interval DEA method, in which the efficiency value of DMU is interval number. In the actual evaluation and selection of bank efficiency, the input and output data are not only accurate values, but also interval values due to the selected index attributes, incomplete information and the need for prediction.

\section{Conclusions}

The existence of unexpected output is an unavoidable fact. For any one of the fixed significance level value and the expected efficiency value, the optimal value increases with the increase of the other index, which provides more effective information for decision makers to evaluate decision units in practice. For all the existing DEA models that consider unexpected factors, the index values of the unexpected factors considered in the model must be accurate, which will inevitably make those models less practical. This paper establishes a two-stage DEA cross-efficiency bank efficiency evaluation model with dual role variables based on unexpected output factors. The evaluation and selection of bank efficiency in actual production, because of the uncertainty of some evaluation indexes or the uncertainty of evaluation indexes caused by external factors, the obtained evaluation index value is usually an interval value besides the accurate value. The new model can evaluate the overall efficiency of the decision-making unit with multi-stage production process when unexpected output occurs, and decompose it to obtain the efficiency value of each stage, thus evaluating the performance of each stage.

\section{Acknowledgement}

This paper was supported by the National Natural Science Foundation of China, "The prevention, control and release of unsafe emotion for high-risk industry employees: An empirical study based on affective events management, emotional tolerance capacity and emotional carrying capacity" (Grant No. 71573086).

\section{Reference}

1. Wang Meiqiang, Li Yongjun. Evaluation of suppliers with fuzzy inputs and outputs-Based on DEA game cross efficiency method [J]. Industrial Engineering and Management, 2015 (1): 95-99.

2. Zhao Jing, Yang Yuzhong, Gao Xuehong, et al. Multi-level supplier evaluation model based on CS-DEA and GRA [J]. Logistics Technology, 2016, 35 (11): 55-60.

3. Wang Meiqiang. Evaluation of suppliers with undesired output factors in fuzzy environments [J]. Statistics and Decision, 2019 (16).

4. Jiang Tianhong. Performance evaluation of electronic manufacturing suppliers based on SE-DEA method [J]. Zhifu Times, 2017 (4X): 255-255.

5. Yan Yi, Feng Xinhai, Tang Zhenzhen. A comparative study of the performance of ETF and other open-end funds based on DEA model [J]. Journal of Hebei University (Philosophy and Social Sciences Edition), 2019, 44 (3): 51-60.

6. Zhao Yue. Evaluation and analysis of water resources utilization efficiency based on DEA model [J]. Urban Geography, 2016 (20): 124-125.

7. Bai Yangmin, Peng Yubing, Cui Ting. Evaluation of enterprise technological innovation capability based on AHP-DEA model [J]. Statistics and Decision, 2015 (12): 169-171.

8. Liu Qi. Performance evaluation of private education financing based on DEA model [J]. Accounting and Communications: Comprehensive (in), 2015 (3): 20-22.

9. Gao Changyuan, Shi Xiaowei, Peng Dinghong, et al. DEA efficiency evaluation model for a class of ring production system $[\mathrm{J}]$. The only official website of computer engineering and application, 2015, 51 (13): 251-254.

10. Du Shi, Zhang Qi. DEAHP-based regulatory operation efficiency evaluation model [J]. Aeronautical Computing Technology, 2015 (1): 22-25. 